\title{
ON THE COMPARISON OF FILTER MAGNETOGRAPHS AND THE ADVANCED STOKES POLARIMETER
}

\author{
HAROLD ZIRIN \\ Big Bear Solar Obs., California Inst. of Technology, Pasadena, CA 91125
}

(Received 12 July, 1994; in revised form 31 March, 1995)

Abstract. Lites et al. (1994) (hereafter LMS) have recently published a comparison of the response of their magnetograph (the Advanced Stokes Polarimeter (ASP)) to that they expect from filter-based magnetographs (FM). Not surprisingly, they conclude the ASP is better. They claim that only their instrument is "quantitative," and others are not, and that the transverse field strength and azimuth measured by filter magnetographs may be in error by up to 50\%. While the calculation is formally correct it ignores the high sensitivity attained by real FM's accumulating thousands of difference frames. Further, FM's have been cross-compared and tested empirically, without any such errors appearing. We point out that the two instruments have different roles, but the functional use of the FM is far superior to the ASP for solar research. The ASP may give accurate results for unresolved unipolar fields; it gives totally erroneous results when the field rapidly changes direction within its resolution element, as occurs in delta spots.

While the ASP is no doubt a powerful instrument for its purpose, the comparison with the FM has to be made on a realistic basis. The two instruments have different roles and virtues: the ASP measures four Stokes parameters simultaneously, while the FM expects the parameters not to change in a minute or so. But the ASP must match up slit after slit in its spectroheliograph mode, degrading spatial resolution. So each has an advantage. Lites et al. base their analysis on a single set of spectra analyzed two different ways, one with their normal fitting routine and the other limited to the spectral band that might be used by an FM. They compare the results, assume the ASP to be perfect, and attribute discrepancies to errors in the FM. They proclaim their instrument the only "quantitative" one and accuse the FM of errors up to 50\%. While the venue of their observations is not specified by LMS, we believe it is the tower telescope at SPO. They apparently do not compare to a real FM, but to the result given by a similar measurement on the ASP data. However, the "extensive calibration" is wholly internal; the only empirical calibration given is for $V$ data only with Rabin's (1992) IR measurement, quoting a divergence of $20 \%$ (the details of that comparison are not published anyway, but we know Rabin only had V data). There is no external calibration of transverse fields. Further, it is well known that magnetic elements occur in "gaps" in the spectral line where the profile is poorly known. This limits the accuracy of any magnetograph.

There are severe shortcomings in the LMS comparison. They do not address the signal/noise ratio $(\mathrm{S} / \mathrm{N})$ of the spectra as compared to the filtergrams, the better spatial resolution of the FM, and the advantage gained by a real FM in accumulating many frames. For example, Figure 1, which is far more sensitive than any data ever published from ASP, is the result of 2048 pairs. This gives S/N 45 times higher than the LMS estimate. The FM produces a highly photometric image of the field in a not-so-well defined spectral band, while a spectrographic magnetograph gives a noisy measurement of a well-defined spectral region along a slit whose position is ill-defined. Image jitter 
of the ASP can lead to distortion of such derivatives as shear and currents. For the steep field gradients of maximum interest these effects can be large.

Resolution is important; the FM averages over a resolution element, and while it measures the flux correctly, it can only underestimate the peak field. LMS use their solutions to estimate the filling factors, which then are considered part of the FM error. With the ASP large splittings may be detected in the profile, even if the element is not resolved. This difference is somewhat alleviated by the higher spatial resolution of FM, which reduces the filling factor. Further, it is not correct at all for fields that reverse sign within short distances. The spectroheliograph-type spatial resolution of the ASP is always inferior to the FM, and the ASP system would have no hope of resolving rapid spatial or temporal changes. Opposite polarization cancels and totally spurious results are obtained for unresolved field reversals. Zirin and Wang (1993a) showed transverse fields alternating in direction in a few arc sec. This extreme variation that occurs in the most interesting delta spots would simply be missed by the ASP. This effect can be seen in the moving magnetic features in LMS Figure 7; such features are normally bipolar under high resolution (Zirin and Wang, 1991), but are smeared to unipolar elements by ASP. A ruler applied to that figure reveals that the resolution is more like 2 or 3 arc sec rather than the sub-arc-second claimed.

The resolution problem can probably not be removed for either instrument. But the accuracy of measurement of either can be determined: random errors may be found by comparison of magnetograms taken in quick succession, and scale errors, by intercomparison with other measurements, such as direct Zeeman splitting. While LMS claim reproducibility they give no data on the variation of successive ASP measurements. This is easy to get from an FM, which takes many repetitive frames. To evaluate scatter in our best VMG data, I have measured the variation between Figure 1 and a magnetogram taken 7 min later. The rms difference between the two magnetograms of the signal per pixel is 148 , and the average signal, 819 . Thus the $S / N$ is about $5.5: 1$ for an 0.5 " squared pixel, and a $2 \times 2$ arc sec box gives an accuracy of 5\%. This is an upper limit, because the intrinsic fields may have changed. For the sensitivity I used a second method (Wang et al., 1985), where we measure the flux of the weakest elements present on both magnetograms. This was 10 gauss over $1 \times 1$ arc sec, a minimum detectable flux of $5 \times 10^{16} \mathrm{Mx}$. LMS claim, without evidence, a high sensitivity; but they have never published quiet Sun fields, which are routinely measured by the FM. What we have seen appears to be at least 10 times less sensitive than the best FM data.

Intercomparison (Wang et al., 1992) among three FM stations showed agreement around $20 \%$ above $1200 \mathrm{~g}$. Variations in instruments, seeing, procedures and times of observation increase the apparent differences; the actual error must be less. Varsik (1994) analyzed the calibration of the BBSO FM, the videomagnetograph (VMG), finding a standard deviation of $14 \%$ over a two-year period. This is the change in the calibration constant; the calibrated data is of course more accurate. The open-loop calibration by Varsik is indeed subject to error in network and plage because of the known variation of profile in the gaps. However Varsik closed the loop by comparing those results with measured line splittings, finding surprisingly good agreement. Thus the open literature establishes that the absolute V calibration of the FM is as good as or better than the $20 \%$ claimed by LMS, who also furnish no data on frame-to-frame fluctuation of their instrument. 
LMS complain that there is no published data on the derivation of vector fields from the FM. But Varsik (1994) has discussed that procedure and its errors. Varsik compared FM vector data with penumbral splitting measurements and found a standard deviation of 180 gauss, $10-20 \%$ of the measured value. These are true empirical calibrations. Thus even the uncalibrated data is accurate at the level of the ASP. This may be because the VMG is mounted on a polarization-free straight-through telescope, while the SPO tower must be corrected. Most published data on azimuths consist of pictures of radial arrows from round spots; the real test is measurement of complex sheared fields. As usual, there is no scatter data for the ASP. For the FM, Wang et al. (1994) show the azimuth varying by less than 5 degrees over hours in a search for flare-connected azimuth changes. The only data I know of for non-radial vector fields compared to Zeeman patterns was published by Zirin and Wang (1993b).

LMS state that FM transverse field directions can be in error by $50 \%$. That may be true for the SPO tower, but has never been seen for real FM. This is easy to check by examining FM data for plages. In a normal plage, the magnetic field is radial. If we observe it some distance from disk center, a transverse field pointed toward the limb appears. Any deviations in a normal plage would be in error. We have never found such errors in our data or that of others and LMS do not actually cite any. Crosstalk between $\mathrm{V}$ and $\mathrm{Q}$ can also be checked this way. LMS have not shown such errors in published data, nor have they shown that theirs is free from it.

One also can measure the elevation of the field exactly by measuring the apparent reversal (Zirin, 1991) of the V signal when the longitudinal projection of the field changes from toward us to away. At that point the field lies in the plane of the sky, and, except for the azimuth in that plane, is fully determined.

In every application, different field properties are important, and the instrument should be appropriate. For studies of activity and field evolution, absolute field strength is of interest, but it is the spatial distribution and temporal evolution that count. This is the intent of the FM systems. The ASP appears designed to analyze the sunspot structure at a single point in time, and appears to do that well. To study Evershed flows, network evolution, and flare-related changes, decent time resolution ( $<4 \mathrm{~min})$ and long sequences (50-100 frames) are necessary. The instrument should always be ready or running, and data must be available in real time to permit necessary adjustments.

The most challenging problem of all is to detect vector field changes in solar flares. This requires stability and high time resolution. The most important application is the search for temporal field changes associated with flares. The FM's at Huairou and BBSO have now detected field changes in six flares (Wang et al., 1993), and the MSFC system, a few more. It would have been impossible to do the round-the-clock comparisons with Huairou if the azimuths were subject to big errors. Wang et al. plot the shear angle of the field before and after several flares. It varies by less than a degree in 5 hours before the flare, changes abruptly by 5 deg during the flare, then remains stable again. This shows the importance of stability, which has been established for the VMG.

To study the evolution of quiet Sun and polar fields, both sensitivity and stability are important. The BBSO VMG, an FM system, detects the intranetwork fields, even the polar fields, which are reduced by the cosine of the central distance. The ASP data that have been published are strictly for sunspots and plages; the system cannot make time sequences or detect intranetwork fields or the crucial polar fields. Not only does Figure 
1 show extremely weak fields, but 100 magnetograms of similar quality and more of lesser quality were obtained on the same day. Transverse fields are unimportant for the quiet Sun, but high resolution enables us to resolve the reversal of field in canopies. Studies of the evolution of the network, XBP's, and the polar field are only possible with the VMG.

The FM and the ASP are such complex instruments that the only sensible comparison is head-to-head observation of the same fields. Coordinated campaigns would be highly desirable, both for intercomparison calibration and recording as much magnetic data as possible. This would also take advantage of the different abilities of each. Claims on resolution and sensitivity are easy to make, but should be substantiated by successive frames, magnetograms of weak-field targets and objective spatial scales.

This work was supported by NASA under NAGW-1972 and NSF under ATM9320822. I am indebted to Drs. Wang and Varsik for important conversations.

\section{References}

B. W. Lites, B. W., Martinez Pillet, V. and Skumanich, A.: 1994, Solar Phys. 155, 1. Varsik, J.: 1994, BBSO preprint 0361, Solar Phys., accepted.

Wang, H., Varsik, J., Zirin, H., Canfield, R., Leka, K. D. and Wang, J.: 1992, Solar Phys. $142,11$.

Wang, H., Ewell, M. W., Jr., Zirin, H. and Ai, Guoxiang: 1994, Astrophys. J. 424, 436.

Wang, J., Zirin, H. and Shi, Z.: 1985, Solar Phys. 98, 241

Zirin, H. and Wang, H.: 1991, COSPAR Proceedings, Adv. Space Res. 11, (5)225.

Zirin, H. and Wang, H.: 1993a, Nature 363, 426.

Zirin, H. and Wang, H.: 1993b, Solar Phys. 144, 137.

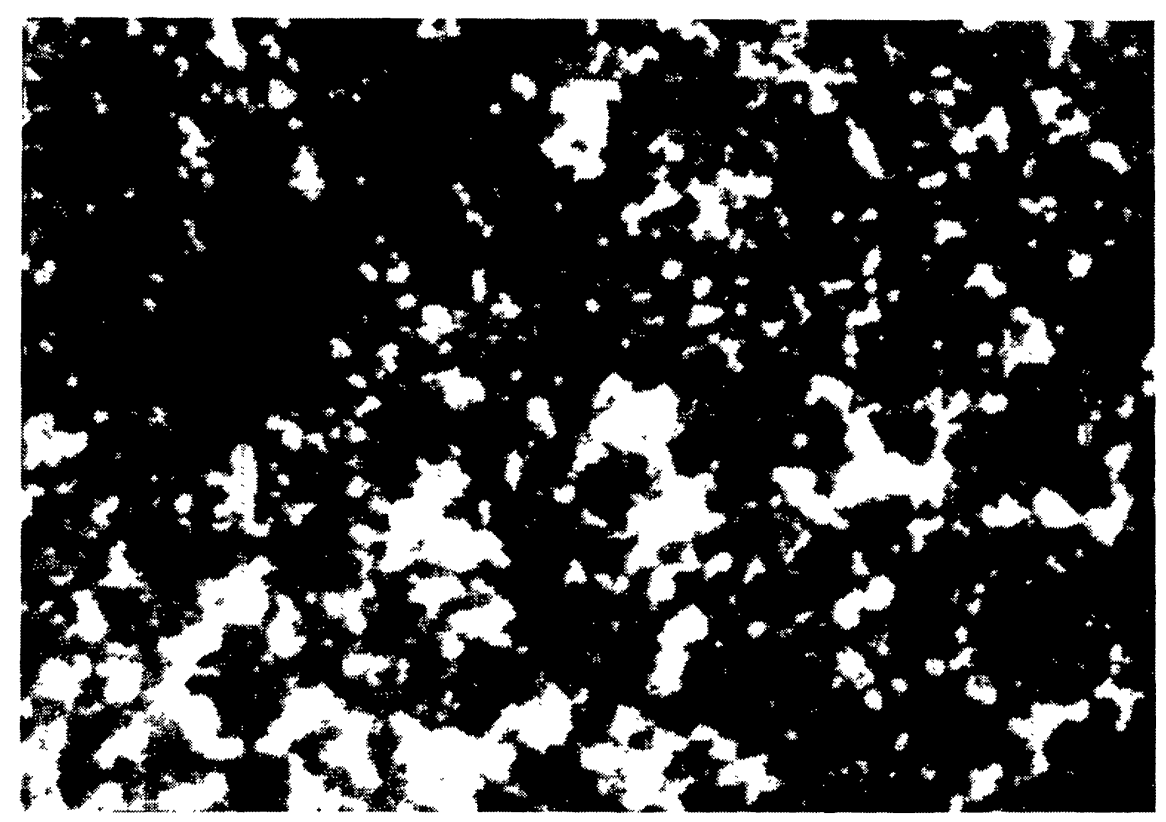

Fig 1. A videomagnetogram of the quiet Sun in $~ 6103$ taken at BBSO June 4, 1992, averaging $2048 \mathrm{~V}$ pairs. The threshold sensitivity is 10 gauss for a $2 \times 2$ pixel area; each pixel is 0.5 arc sec. The print enhances the weaker and saturates the stronger field. The horizontal field of view is 200 arc sec. 